\title{
Using IQA to Extract Mental Models Concerning Quality Evaluation Factors for Social Networking Services on Smartphones
}

\author{
Yu-Lou Lee', Li-Chieh Chen² \\ ${ }^{1}$ The Graduate Institute of Design Science, Tatung University, Taiwan \\ ${ }^{2}$ Department of Industrial Design, Tatung University, Taiwan \\ Email: carry19830331@hotmail.com
}

Received 23 November 2015; accepted 3 January 2016; published 6 January 2016

Copyright $(\underset{2015}{ }$ by authors and Scientific Research Publishing Inc.

This work is licensed under the Creative Commons Attribution-NonCommercial International License (CC BY-NC).

http://creativecommons.org/licenses/by-nc/4.0/

(c) (i) Open Access

\begin{abstract}
Although today's users of mobile phones are afforded increasing control as the popularity and richness of information provided by smartphones increases, how these users make choices and evaluations cannot be explicitly expressed. This study uses an acute observation of designers of technology and aesthetics to conduct a mental-model analysis of smartphones with the purpose of explicitly identifying the differences in the schemas of users. This study also modifies the mental model to evaluate the practicability of the mental model. Hence, this study investigates groups of subjects, comparing freshmen and graduating students froma college of design, and adopts a schematized data collection and analysis approach, Interactive Qualitative Analysis (IQA), to ascertain the relationships between factors and establish a complete user mental model. The study results present 11 primary affinity factors: "Pricing," “Advertisement," “User Interface," "Innovation Functions," "System Maintenance," "Privacy," "Utility Functions," "Personalization," "Network Performance," "Customer Service," and "Multimedia Contents." The primary driver in evaluating smartphones is found to be "Pricing" for graduating students and "Privacy" for freshmen. The two groups share the same final effect factor, "Multimedia Contents." This study has successfully identified differences between the different mental models of the two groups, supporting the method that using IQA to perform a quality evaluation of social networking for smartphones is effective. Consequently, platform developers can understand user demand through a mental model and can design good platform functions to effectively improve users' experience of smartphones.
\end{abstract}

\section{Keywords}

Interactive Qualitative Approach, Smartphones, Social Networking Service (SNS), Mental Model 


\section{Introduction}

As the technology of mobile phones develops, the richness of information and platform interaction provided by mobile phones play an increasingly indispensable role in life, with the result that users now have more autonomy and choice. However, what thought process do users choose and determine things? Johnson-Laird mentions that the human mental model is related to affinities with the outside world [1]; when we interact with something, we form an internal mental model of certain interactions and apply it when predicting and explaining new situations or problems [2]. Norman also suggests that users understand the overview of the products through an internal mental model [3]. It is suggested that the internal mental model is acquired from prior knowledge and experience. The mental model is the perspective through which people view things and is also a cognitive schema diagram; different people tend to have different cognitive schema toward the same thing. For this reason, the study adopts the Interactive Qualitative Approach (IQA) to understand the internal evaluation standard of mobile-phone users and to analyze the mental model of smart phone users. This study also applies the mental modelsit derives from the freshmen and graduating students in order to prove the difference between the two and to evaluate whether IQA can be applied to analyze the practicability of the mental model of smartphone users. This paper is organized as follows: First, the paper will present literature reviews concerning social networking services for smartphones, user experience, mental models, and the definition of IQA. Second, the paper provides hypotheses and the procedure. Third, the results of the interrelationship diagram (IRD) of mobile-using graduating students and the mind map for these students are discussed. Finally, the authors discuss the findings, summarize their conclusions, and make recommendations for future research.

\subsection{Social Networking Services for Smartphones}

The key success to the development of smartphones is the integration of smartphones and wireless Internet, which has resulted in a rich flow of information and changed the way people communicate as well as their lifestyles. Public demand for information is on the rise, suggesting that mobile phones are not used passively but have become anew interactive model of transmission and communication. Nonetheless, different concepts will be formed as the information trends in society develop toward smartphones. Recent studies conducted by scholars demonstrate two categories after analysis. The first category is the value of a positive user. For example, Park and Han conducted a study on the value of user experience by analyzing factors such as convenience, pleasure, aesthetics, and friendship to determine if users are satisfied with their own smartphones [4]. Kim and Han applied online advertisements, traffic, and personalization to analyze why advertisements of smartphones attract users [5]; the study results suggest that personalization could be seen as one of the factors that encourage purchase intent. Moreover, purchase intent increases as the flow of experience and reliability, entertainment, and advertising incentives increase. Fazal-e-Amin discovered through analyzing the user characteristics of web browsers on smartphones that there was no significant difference between different age groups in relation to browser use [6]. Kim et al. analyzed the innovation and application purposes of smartphones based on differencesin social demography and personality, suggesting that young, educated, wealthy, open, extroverted, and rigorous people tend to use smartphones and their applications more. The second category refers to the negative impact [7]. A study conducted by Lee et al. suggests that the compulsive use of smartphones has a certain impact on psychological control, social anxiety disorder, material desire, and the desire for physical contact [8]. In their study exploring the privacy and security of smartphones, Jones and Chin discover that users tend to put their security at risk by exposing personal information when using the mobile phones for financial management [9]. Chiu proposes in his study that social efficacy and stress from family and emotions are susceptible to exacerbation through the use of mobile phones [10]. Van Deursen explored the customs, emotional quotient, social stress, self-modulation, gender, and addictive conduct in relation to age in society-oriented use of smartphones [11]. The study reveals that women are more likely to develop addictive habits when using mobile phones while older people are less likely to develop addictions to mobile phones. Fully considering the above body of research, we can see that everyone perceives and uses smartphones differently, whether for positive use or negative impact. It is suggested that only the explicit identification of the distinctive opinions and requirements of users can effectively help smartphone developer steam prove user experience and design smartphones that meet users' requirements.

\subsection{User Experience}

"Experience" is the key to consumer behavior [12], and good cognitive interaction aims to create successful user 
experience, such as when Mishra evaluated user experience through advertisements on Facebook, suggesting that user experience is related to good design [13]. User experience refers to users' perception of a specific product, system, or interactive process. Hassenzahl \& Tractinsky mentioned that user experience is a complex and dynamic subjective concept and also represents an interaction between the intrinsic and extrinsic systems of users [14]. Consequently, different users can hold different expectations and, as a result, perform different uses and hold different opinions [15]. Schmitt proposed the "experience construct," for which he received inspiration from the interaction of perceived contact, conscious contact, and cognitive systems through the spiritual module [16]. Through this, he established the psychological experience construct of users and divided the experience into five constructs: the "sense construct," "emotion construct," "thinking construct," "action construct," and "correlation construct." To provide excellent experiences and produce profound impressions on users, a variety of experience forms should be implemented interactively by requiring the users to experience these constructs to achieve objectives. Such a process can provoke intrinsic reactions. Moreover, the aesthetic experience must attract users by providing them with satisfaction derived from psychological effects and allowing them to accomplish emotional identification, thereby forming a scheme of personal judgment standards. Finally, the users are left with a profound and touching experience to share with others.

\subsection{Mental Model}

To comprehend the experiences perceived by users, a user-oriented mindset is required to identify the relationship between people's overviews, the system, and the environment [3]. An analysis of studies related to mental models reveals that the mental model is a rich and sophisticated cognitive framework, constructed within the context of our experienced situations [17]. A study conducted by Sanchez to analyze the social techniques of virtual social networking using IQA reveals result [18], where the mental model is the result of composite mental operations. Hence, in order to understand the composition of users' mental models in-depth, mental models for users' experiences of smartphones should be taken into consideration.

Internal mental models are acquired from prior knowledge and experience, but the development ofproficient knowledge and experience takes considerable effort. The study conducted by Katz proposes that it takes 3 - 5 years of teaching for novice teachers to enter the proficiency stage [19]. Contrarily, Huberman proposes that it takes 4 - 6 years to enter the stable-expert stage [20]. So, could the difference inknowledge between novice and proficient levels affect the selectionof requirements for smartphones and thereby present differingmental models? Could a mental model be applied to precisely determine the ideas of users? This study's subjects are comprised of freshmen and graduating students from the Department of Design with an emphasison technology and aesthetics. Freshmen and graduating students acquire different knowledge during different learning stages in university; graduating students inevitably have a higher level of professional knowledge than freshmen after years of schematized professional learning. Hence, Stage 1 and Stage 2 of this study emphasize the different determinations of schemas for smartphones in freshmen and graduating students. Additionally, through the use of IQA, these stages analyze the differences in the mental models between the two groups created by different choices relating to smartphones.

\subsection{The Definition of Interactive Qualitative Analysis (IQA)}

The IQA approach is a systematic qualitative research method developed by Northcutt, Miles et al. in 1998 [21]. IQA is an analytical method for conducting structured and systematic qualitative analyses and has already been employed in various fields [22]. It uses focus groups comprised of subjects with similar backgrounds, in which brainstorming and interviews can be conducted. By sharing their individual thoughts and experiences, people can resolve the causal relationship between subjects' deeply held experiences and their conceptualizations [23], thereby obtaining better needs-assessment factors [24]. The IQA research method aims to gather subjects who have a good understanding of the research topic and who will share their thoughts through their feelings and experiences. Through the consistency, coherence, and structure of the group's views, people can then find the concept within a topic and find a causal relationship between the topic and the concept [25], thereby creating a mind map. The IQA research method can clearly present causality of concepts through the use of mind maps [21]. Finally, this research improved upon the IQA model by using individual detailed interviews and by introducing questions without a structure, causing the subject to express their thoughts and opinions purely in response to the research topic. This research used a questionnaire in addition to an interview, consequently ac- 
quiring more standardized and richer insights. The IQA method is a systematic qualitative method that considers the subjects' understanding of complete coding and mind maps and, therefore, is suitable for this paper's study of smartphone users' mind maps.

\section{Method}

\subsection{Objectives}

This study selected participants from freshmen and graduating students in the Department of Design. The designers apply professional knowledge of aesthetic design, acute observation, and boldness in creating new tech gadgets, occupying the front-line of development at a time when smartphones are one of the products of newgeneration technology integration. Designers conduct smartphone mental model analysis using sensitivity to technology and aesthetics, thereby evaluating the effectiveness of the mental model in evaluating new gadgets. The freshmen and graduating students from the department of design comprehend the difference of requirements with the purpose of clearly identifying the difference in users' schema, resulting in demand differences and forming different mental models, thereby determining the practicability of applying mental models to smartphones.

\subsection{Hypotheses}

1) Is the mental model obtained through IQA applicable to perform quality evaluation forthe measurement of social networks for smartphones?

2) Can the mental model obtained through IQA be used to explicitly identify the difference between different groups in the use of smartphones?

\subsection{Procedure}

Through a focus group approach, Stage 1 of thisstudy considers the characteristics shared by the graduating studentparticipants that cause individuals to choose smartphones. This approach acquires the real viewpoints and determinants of respondents to the research topic. Stage 2 applies the use of questionnaires and the interviews conducted with freshmen respondents, using the concepts derived from the focus-group approach with the graduating students for the questionnaire concept interrelation diagram to conduct in-depth individual interviews to ascertain differences and similarities in affinities.

Stage 1 of this study is based on the mental model of communication experience for smartphone users, adopting a schematic data collection and analysis approach: an Interactive Qualitative Analysis Approach (IQA). The analysis process through IQA explicitly reveals the causality between concepts [26].

Stage 1: First, we separated our sample of smartphone users into groups of freshmen and graduating students. For the focus group of graduating students, after providing them with the purpose and method of the research, they were asked to write on cards words that they associate with the research topic. This step was designed to allow the seniors to clearly describe their points of view while the researchers proposed questions. After all of the graduating students had received clarifications, they were asked to carefully consider these questions without discussing with each other. Participants were encouraged to provide and fully describe as many relevant thoughts or experiences as they wished. At this time, students were expected to attempt to write approximately 25 cards during a silent brainstorming session lasting one hour. After the students had completed writing, all of their cards were arranged, and the participants were asked to observe them. Students could then discuss the similarities among the cards, and rearrange thecards to place those with similar meanings together. At this point, researchers could direct the students in assigning a name to each group of similar cards, with the name being derived from its applicability to nouns of similar meaning. After confirming with students and clarifying and correcting in order to achieve a consensus, each of these names was called an "affinity." Using these affinities to create an Affinity Relationship Table (ART) for the questionnaire, and giving it to the seniors to complete, an IRD could be organized accordingly.

In Stage 2, the study improvedupon the traditional approach by using only anART forthe second group that was generated from in-depth interviews with individuals. The study adopted the affinity factorschosenby graduating students to createa questionnaire ART and requestedthat the freshmen complete the ART. The study applied simple analysis to compare the differences of ART between graduating students and freshmen, followed 
by conducting individual interviews with the freshmen on the difference and similarity in affinities, there by assisting the understanding ofdifferencesinmental determination and sorting in the new IRD. The study then analyzed the internal views and affinities of graduating students and freshmen using the respective IRDs in addition to drawing two system-influence diagrams (SID) to comparethe correlation between factors. A complete mental model for graduating students and freshmen wasestablished throughthe research operation steps described in Figure 1.

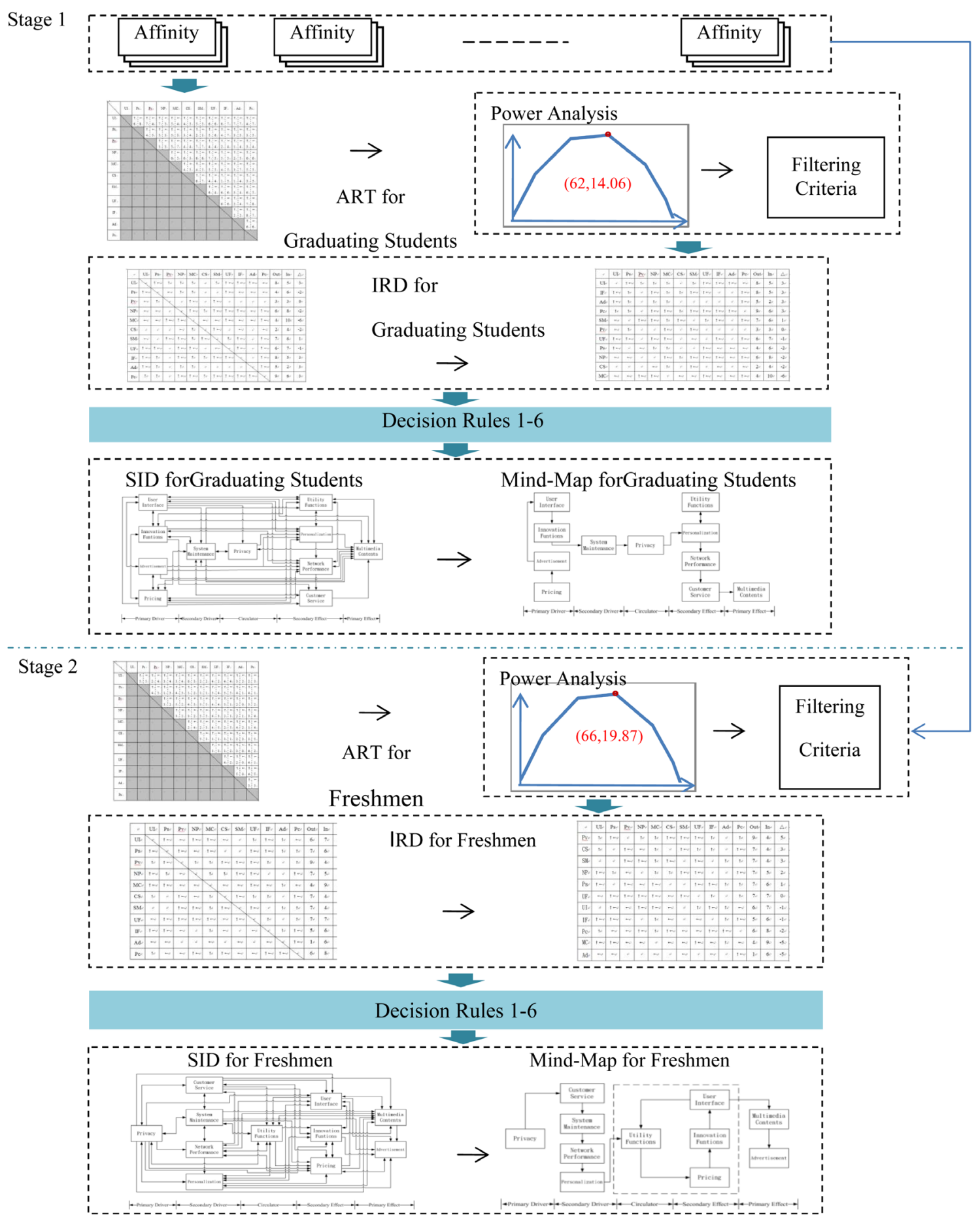

Figure 1. Mental model extraction experiment. 


\section{Results}

\subsection{Interrelationship Diagram of Mobile-Using Graduating Students}

The study adopts IQA to extract 11 affinity factors from the graduating students, including User Interface (UI), Personalization (Ps), Privacy (Pv), Network Performance (NP), Multimedia Contents (MC), Customer Service (CS), System Maintenance (SM), Utility Functions (UF), Innovation Functions (IF), Advertisement (Ad), and Pricing (Pc). These 11 affinity factors form the ART, which wasgiven to the eight graduating students to complete. The ART of these eight graduating students is consolidated while the frequency of the appearance of each affinity relationship is sorted in descending order according to the 80/20 rule or the Pareto principle. The 110 interrelationships yield to 524 results, which are then used to calculate the cumulative frequency rates, cumulative percentage relations, cumulative percentage frequency rates, and explanatory powers. The study then applies the MiniMax Ratio proposed by Young [27], using the MiniMax decision approach where there isuncertainty, to express the maximum variance as standard using the least affinity quantity, thereby determining which affinity interrelationship can be incorporated into IRD. The study results show that at the $62^{\text {nd }}$ relationship (system maintenance $\leftarrow$ Pricing related), the explanatory power reaches amaximum value of $14.06 \%$ while the cumulative percentage frequency rate reaches $70.43 \%$, as shown in Figure 2 . The frequency rate at this time is five and only the affinity relation of "frequency rate $\geq 5$ " is used to construct the interrelationship table of graduating students, as shown in Table 1.

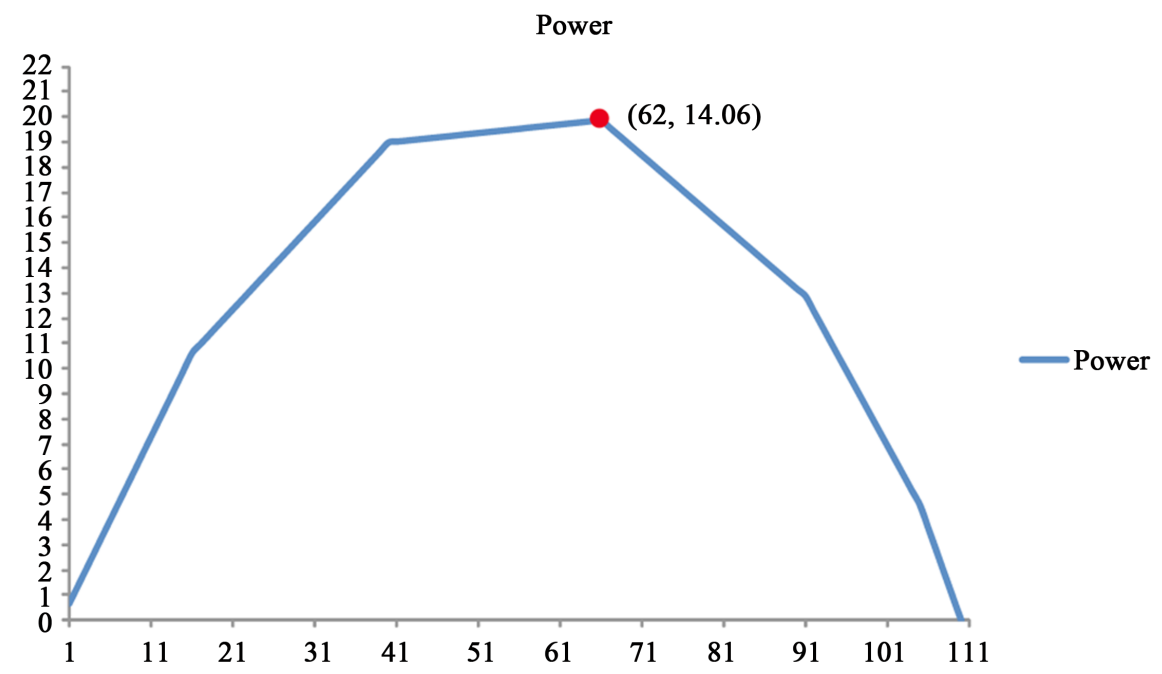

Figure 2. Run chart for changes in explanatory power.

Table 1. Graduating student interrelationship diagram.

\begin{tabular}{ccccccccccccccccc}
\hline & UI & Ps & Pv & NP & MC & CS & SM & UF & IF & Ad & Pc & Out & In & $\triangle$ \\
\hline UI & & $\uparrow \leftarrow$ & $\uparrow$ & $\uparrow$ & $\uparrow$ & & $\uparrow$ & $\uparrow \leftarrow$ & $\uparrow \leftarrow$ & $\uparrow \leftarrow$ & $\leftarrow$ & 8 & 5 & 3 \\
IF & $\uparrow \leftarrow$ & $\uparrow$ & & $\uparrow \leftarrow$ & $\uparrow$ & $\uparrow$ & $\uparrow \leftarrow$ & $\uparrow \leftarrow$ & & & $\uparrow \leftarrow$ & 8 & 5 & 3 \\
Ad & $\uparrow \leftarrow$ & $\uparrow$ & & $\uparrow$ & $\uparrow$ & & & & & & $\uparrow \leftarrow$ & 5 & 2 & 3 \\
$\mathrm{PC}$ & $\uparrow$ & $\uparrow$ & & $\uparrow \leftarrow$ & $\uparrow \leftarrow$ & $\uparrow$ & $\uparrow \leftarrow$ & $\uparrow \leftarrow$ & $\uparrow \leftarrow$ & $\uparrow \leftarrow$ & & 9 & 6 & 3 \\
$\mathrm{SM}$ & $\leftarrow$ & & $\uparrow \leftarrow$ & $\uparrow \leftarrow$ & $\uparrow$ & $\uparrow \leftarrow$ & & $\uparrow$ & $\uparrow \leftarrow$ & & $\uparrow \leftarrow$ & 7 & 6 & 1 \\
$\mathrm{Pv}$ & $\leftarrow$ & $\uparrow$ & & & $\uparrow \leftarrow$ & & $\uparrow \leftarrow$ & & & & & 3 & 3 & 0 \\
$\mathrm{UF}$ & $\uparrow \leftarrow$ & $\uparrow \leftarrow$ & & $\uparrow \leftarrow$ & $\uparrow \leftarrow$ & & $\leftarrow$ & & $\uparrow \leftarrow$ & & $\uparrow \leftarrow$ & 6 & 7 & -1 \\
$\mathrm{Ps}$ & $\uparrow \leftarrow$ & & $\leftarrow$ & $\uparrow$ & $\uparrow$ & & & $\uparrow \leftarrow$ & $\leftarrow$ & $\leftarrow$ & $\leftarrow$ & 4 & 6 & -2 \\
$\mathrm{NP}$ & $\leftarrow$ & $\leftarrow$ & & & $\uparrow \leftarrow$ & $\uparrow$ & $\uparrow \leftarrow$ & $\uparrow \leftarrow$ & $\uparrow \leftarrow$ & $\leftarrow$ & $\uparrow \leftarrow$ & 6 & 8 & -2 \\
$\mathrm{CS}$ & & & & $\leftarrow$ & $\uparrow$ & & $\uparrow \leftarrow$ & & $\leftarrow$ & & $\leftarrow$ & 2 & 4 & -2 \\
$\mathrm{MC}$ & $\leftarrow$ & $\leftarrow$ & $\uparrow \leftarrow$ & $\uparrow \leftarrow$ & & $\leftarrow$ & $\leftarrow$ & $\uparrow \leftarrow$ & $\leftarrow$ & $\leftarrow$ & $\uparrow \leftarrow$ & 4 & 10 & -6 \\
\hline
\end{tabular}




\subsection{Mind Map for Mobile-Using Graduating Students}

According to the interrelationships of graduating students described in Table 1, we aggregated relations 1-62 into an IRD, and calculated the value of Out, In, and $\Delta$. Then, these were sorted in descending order according to the $\Delta$ value and the decision drivers, circulator, and effects were found in order to create the mind map. This research analyzed the $\Delta$ decision rule as follows:

1) Driver if the $\Delta$ value is positive, Effect if the $\Delta$ value is negative, and Circulator if the $\Delta$ value is zero;

2) The largest positive value indicated the group's Primary Driver;

3) Other positive values indicated the Secondary Drivers of the group;

4) The largest negative value indicated the group's Primary Effect;

5) Other negative values indicated the Secondary Effects;

6) Lastly, find the zero value, this is the group's Circulator.

The complex of 62 relations were put together to form a SID for senior students, shown in Figure 3, after simplifying this, a mind-map could be created, as shown in Figure 4.
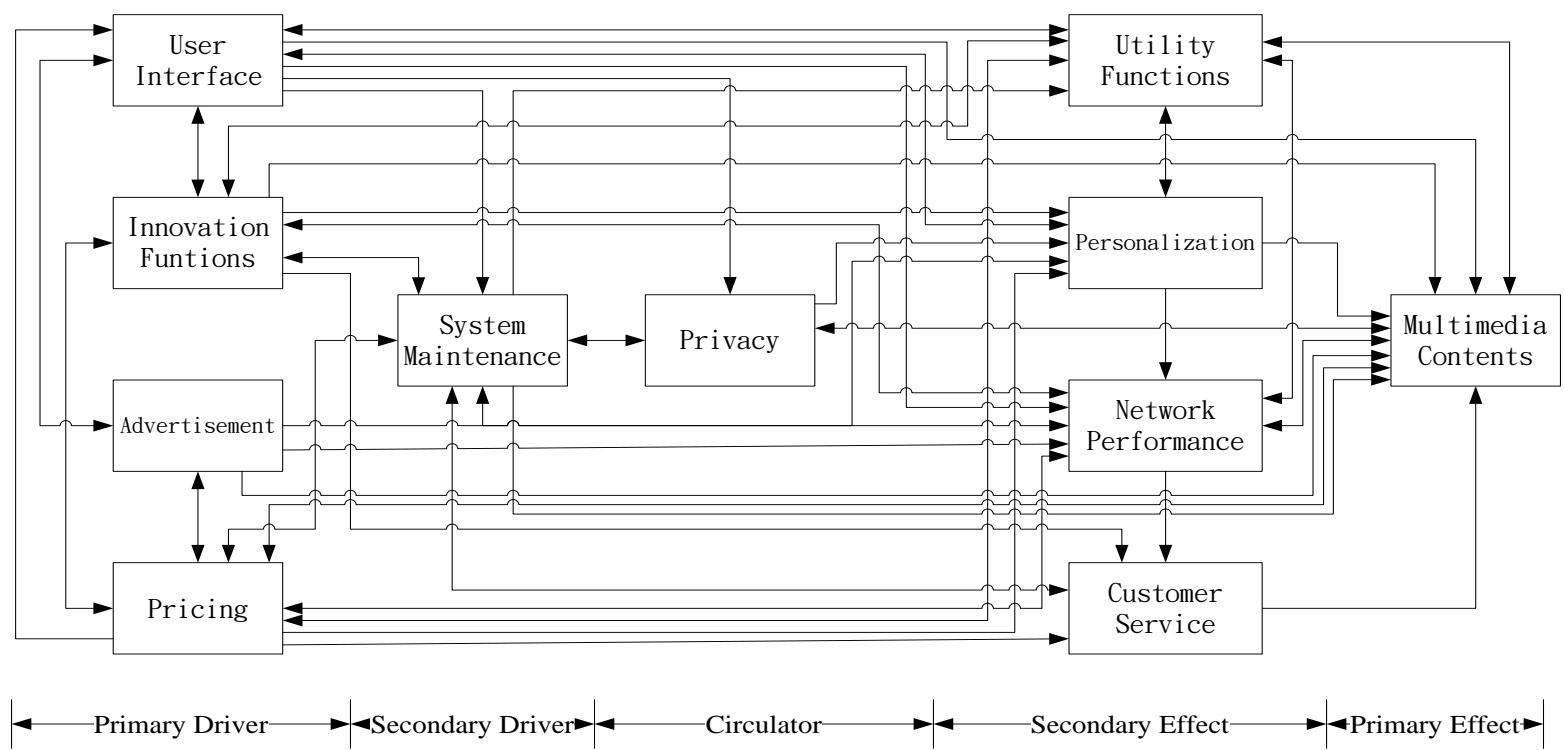

Figure 3. Graduating student system influence diagram.

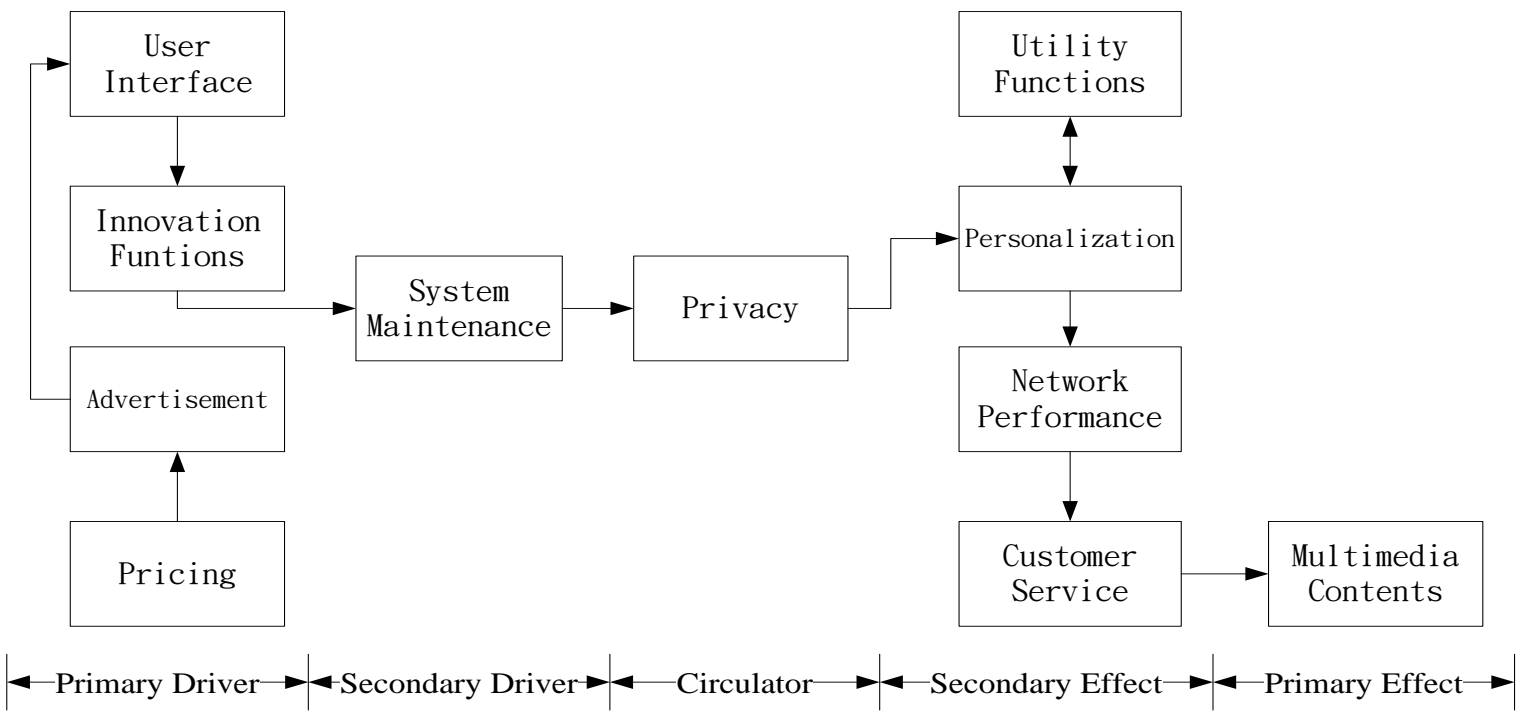

Figure 4. Mind map for mobile-using graduating students. 
The influence affinity path presented through the mind map of graduating students is shown as "Pricing" $\rightarrow$ "Advertisement" $\rightarrow$ "User Interface" $\rightarrow$ "Innovation Functions" $\rightarrow$ "System Maintenance" $\rightarrow$ "Privacy" $\rightarrow$ "Personalization" $\leftrightarrow$ "Utility Functions." "Personalization" $\rightarrow$ "Network Performance" $\rightarrow$ "Customer Service" $\rightarrow$ "Multimedia Contents." The study results show that "Pricing" is the main priority consideration for graduating students. Namely, the price will affect the establishment of interactive advertisements while the amount of interactive advertisement established will affect the quality of interface design, etc. For example, when the price of a smartphone is low, the graduating students believe that the low price will lead to the massive establishment of interactive advertisements since massive interactive advertisements cannot express the quality interface design. The coarse interface design cannot present the innovation function through simplicity. Complex innovation functions intensify the difficulty of system maintenance becausea lack of excellent system maintenance leaves users susceptible to disclosing personal security and privacy. Insecure privacy will affect personalization options, such as users having safety concerns over information services. The respondents are compelled to increase or reduce options for certain information services due to the lack of security in using personalization options, which thereby affects the full function of practicability. Moreover, the speed and performance cannot improve because the personalization options are compelled to increase options for certain information services. This insufficiency in speed and performance will force users to consider additional options for customer services (i.e., upgrading hardware/software equipment). Finally, the additional increase of options for customer service also enhances the diverse presentation of multimedia. It is suggested that the price of smartphones in the evaluation of graduating students will ultimately determine the presentation effects of multimedia diversity.

Therefore, to more precisely understand the viability of applying IQA effectively on the evaluation factors of smartphones, thisstudy provided an additional questionnaire and interviews to apply the affinities of graduating students into the affinity interrelation table. After the freshmen completed the questionnaires, the affinities acquired were discussed and compared to identify the significance ofthe differences when IQA was applied to the different testing groups.

\subsection{Mind Map of Freshmen Smartphone Users}

Next, the 11 affinity factors generated from the graduating students were applied to coding and freshmen participants were required to participate first, with the explanation and description then provided by the researcher. The six freshmen participants completed the personal ART to briefly evaluate the ART of graduating students and the freshmen, followed by a discussion of the difference between graduating students and freshmen. Later, the ART of six freshmen participants underwent a power analysis and the results suggest that at the $66^{\text {th }}$ relationship (advertisement establishment $\rightarrow$ Pricing related), the explanatory power reaches amaximum value of $19.87 \%$, while the cumulative percentage frequency rate is $79.84 \%$. The screening of affinity frequency in freshmen, sorted in descending order, and the 1-62 affinity relations in the explanatory power analysis were consolidated into the IRD with factors summarized. Finally, the IRD wasused to draw the preliminary SID, followed by deleting the repeated paths to generate more simplified new ISD, as shown in Figure 5.

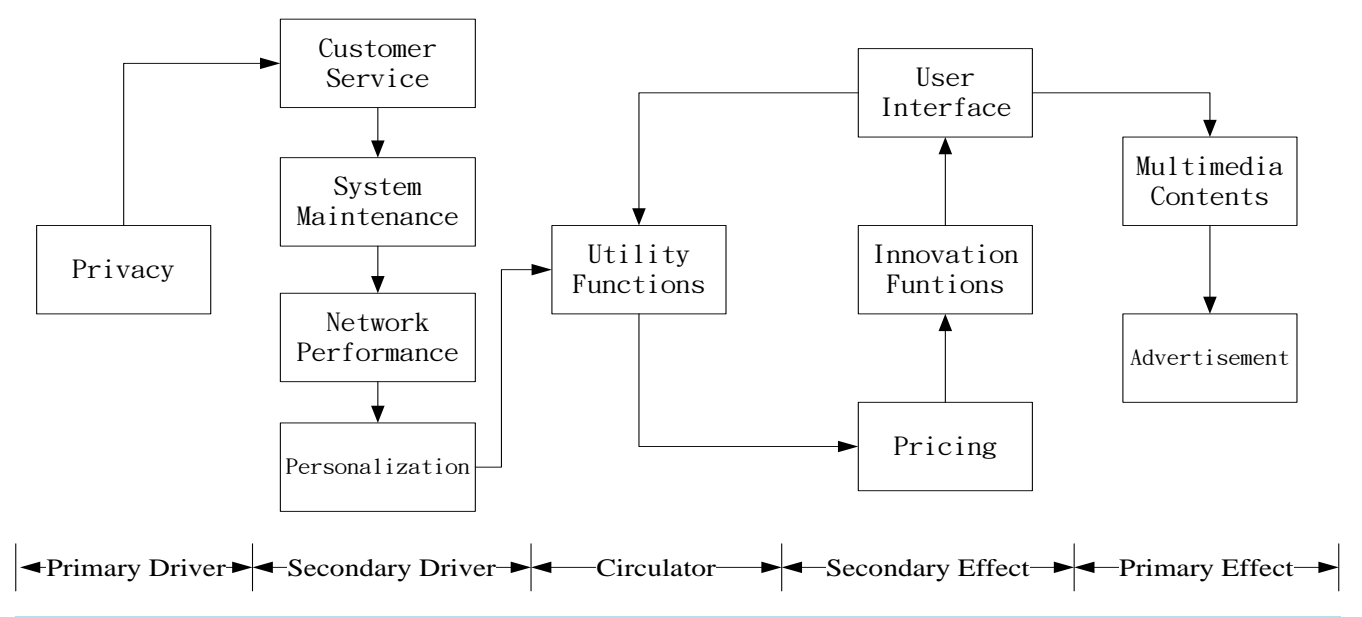

Figure 5. Mind map for mobile-using freshmen. 
The influence affinity path presented through the freshmen mind map is shown as "Privacy" $\rightarrow$ "Customer Service" $\rightarrow$ "System Maintenance" $\rightarrow$ "Network Performance" $\rightarrow$ "Personalization" $\rightarrow$ "Utility Functions" $\rightarrow$ "Pricing" $\rightarrow$ "Innovation Functions" $\rightarrow$ "User Interface" $\rightarrow$ "Utility Functions" and "Multimedia Contents" $\rightarrow$ "Advertisement."The study results show that "Privacy" is the driving factor forfreshmen students in priority consideration. In other words, the level of privacy protection will affect the number of options for customer service while the number of options for customer service will affect the difficulty of system maintenance, and so on. For example, customer service options will increase when there is a problem with the security or privacy of smartphones, which will strengthen the protection. The increase in options for customer services also increases the difficulty of system maintenance, which will inevitably intensify the speed and performance accordingly. The intensity of speed and performance will lead to more options for personalization. The increase of personalization options will expand the functions of practicability while more practicability will relatively increase the overall pricing. Moreover, price increaseswill likely create innovation that requires good interface design for a more user-friendly interface. Good interface design will take consideration of the establishment of practicability and presentation of multimedia diversity. Finally, the presentation of multimedia diversity will affect the approach to establishing interactive advertisements. This suggests that freshmen's evaluation of smartphones'security and privacy protection will ultimately determine the way that interactive advertisements are established.

Moreover, from the simplified mind map forfreshmen, a feedback loop is discovered. The paths are outlined as "Utility Functions" $\rightarrow$ "Pricing" $\rightarrow$ "Innovation Functions" $\rightarrow$ "User Interface" $\rightarrow$ "Utility Functions" (as shown in Figure 3), which means that freshmen users determine the price reasonableness of smartphones through the practicability functions provided by smartphones. This is to verify that the smartphones provide users with default content. In other words, users anticipate that smartphones with higher prices offer innovative and high-quality interface design. Such outstanding interface designs will naturally acquire richer and simpler information services from practicability, thereby forming a feedback loop.

\section{Discussion}

The study modifies the IQA approach and applies it to smartphone users for the evaluation of the effectiveness and practicability of this approach. The results show that both Hypothesis 1 and Hypothesis 2 are supported, where the mental model extracted by IQA can be applied to evaluate the quality of smartphone social networking and effectively discover the difference in different groups' use of smartphones. In view of the overall testing results, the mental model also varies following the accumulation of years of experience and knowledge. The 11 affinities from the mind map of graduating students yield to "Pricing" as the main priority factor for consideration while the students believe that a good smartphone should be equipped with good hardware speed and performance, software maintenance and customer service options, practicability and innovation functions, a dazzling and appealing interface, personalization options, multimedia diversity, valuation on security and privacy, and avoidance of negative advertisement. It is suggested from the choice evaluation of graduating students that the price of smartphones will ultimately determine the presentation effect of multimedia diversity. The results of the mind map for freshmen reveal that supplying a secure smartphone to users will affect the quality of interactive advertising for smartphones. The two study groups initially determine the primary driver for graduating students as "Pricing" and as "Privacy" for freshmen. The cause leading to the two discretions lies with the difference in information capacity. The in-depth interviews with freshmen show that freshmen often encounter problems relating toprivacy and security due to their limited experience with smartphones. The lack of information capacity leads to troubles in the subsequent processing and, hence, privacy and security is the only main driving factor considered by the freshmen in their evaluation of smartphones. On the contrary, the graduating students have used smartphones over a long period time with sufficient information capacity to handle and avoid privacy and security issues. Hence "Pricing" is the primary driver for which graduating students care the most when evaluating smartphones. It is interesting to note that the two groups agree on "Multimedia Content" being the final effect factor. The two groups wish to experience the information richness and enjoy satisfaction from high-quality sound and lighting effects of smartphones, implying that direct visual effects will affect users' satisfaction towards smartphones and thereby determine if theywill continue to use and recommend the mobile phones to others.

When more platform designers attempt to design with consideration for "user orientation,” they are thinking from the perspective of users to identify what the users need and want. This willresult in the designof good platform functions and user experiences. When users enjoy good user experience, they will usually be generous in 
sharing the experiencewithfriends and relatives, giving the opportunity to propagate the phone's positive image successfully. Hence, understanding users' views and demand will effectively accelerate the platform propagation and increase word-of-mouth popularity.

\section{Conclusion and Recommendations for Future Research}

This study used IQA to extract a service quality factor framework of groups of smartphone users. This framework was successful in identifying differences among different groups of smartphone users. In order to verify these experimental results, in the future, the IQA method should be used, employing different participants with different backgrounds, to further our understanding of the decision-making process towards smartphone selection and the viability of the mental model in this respect.

\section{References}

[1] Johnson-Laird, P.N. (1983) Mental Models-Towards a Cognitive Science of Language, Inference and Consciousness. Harvard University Press, Cambridge.

[2] Hu, H.-J. (2011) Exploring the Mental Models of Website Design Team and Internet Users. Ph.D. Thesis, National Yunlin University of Science and Technology, Taiwan.

[3] Norman, D.A. (1986) User-Centered System Design: New Perspectives on Human-computer Interaction. In: Norman, D.A. and Draper, S.W., Eds., Cognitive Engineering, Lawrence Erlbaum Associates, Hillsdale, 31-61.

[4] Park, J. and Han, S. (2013) Defining User Value: A Case Study of a Smartphone. International Journal of Industrial Ergonomics, 43, 274-282. http://dx.doi.org/10.1016/j.ergon.2013.04.005

[5] Kim, Y. and Han, J. (2014) Why Smartphone Advertising Attracts Customers: A Model of Web Advertising, Flow, and Personalization. Computers in Human Behavior, 33, 256-269. http://dx.doi.org/10.1016/j.chb.2014.01.015

[6] Fazal-e-Amin (2015) Characterization of Web-Browser Usage on Smartphones. Computers in Human Behavior, 51, 896-902. http://dx.doi.org/10.1016/j.chb.2014.10.054

[7] Kim, Y., Briley, D. and Ocepek, M. (2015) Differential Innovation of Smartphone and Application Use by Sociodemographics and Personality. Computers in Human Behavior, 44,141-147. http://dx.doi.org/10.1016/j.chb.2014.11.059

[8] Lee, Y., Chang, C., Lin, Y. and Cheng, Z. (2014) The Dark Side of Smartphone Usage: Psychological Traits, Compulsive Behavior and Technostress. Computers in Human Behavior, 31, 373-383. http://dx.doi.org/10.1016/j.chb.2013.10.047

[9] Jones, B. and Chin, A. (2015) On the Efficacy of Smartphone Security: A Critical Analysis of Modifications in Business Students’ Practices over Time. International Journal of Information Management, 35, 561-571. http://dx.doi.org/10.1016/j.ijinfomgt.2015.06.003

[10] Chiu, S.-I. (2014) The Relationship between Life Stress and Smartphone Addiction on Taiwanese University Students: A Mediation Model of Learning Self-Efficacy and Social Self-Efficacy. Computers in Human Behavior, 34, 49-57. http://dx.doi.org/10.1016/j.chb.2014.01.024

[11] Van Deursen, A., Bolle, C., Hegner, M. and Kommers, P.A.M. (2015) Modeling Habitual and Addictive Smartphone Behavior: The Role of Smartphone Usage Types, Emotional Intelligence, Social Stress, Self-Regulation, Age, and Gender. Computers in Human Behavior, 45, 411-420. http://dx.doi.org/10.1016/j.chb.2014.12.039

[12] Arnould, E., Price, L. and Zinkhan, G. (2004) Consumers. 2nd Edition, McGraw-Hill/Irwin, Boston.

[13] Mishra, A. (2015) Consumption Value of Digital Devices: An Investigation through Facebook Advertisement. Social Networking, 4, 51-61. http://dx.doi.org/10.4236/sn.2015.43007

[14] Hassenzahl, M. and Tractinsky, N. (2006) User Experience-A Research Agenda. Behaviour and Information Technology, 25, 91-97. http://dx.doi.org/10.1080/01449290500330331

[15] Tobias, P. and Spiegel, D.D.S. (2009) Is Design the Preeminent Protagonist in User Experience? ACM Ubiquity, 10. http://dl.acm.org/citation.cfm?id=1576100

[16] Schmitt, B.H. (1999) Experiential Marketing: How to Get Customers to Sense, Feel, Think, Act, and Relate to Your Company and Brand. The Free Press, New York.

[17] Carroll, J.M. and Olsen, J. (1988) Mental Models and Human-Computer Interaction. In: Helonder, M., Ed., Handbook of Human-Computer Interaction, Elsevier, Amsterdam, 45-65.

[18] Sanchez, J. (2007) Second Life: An Interactive Qualitative Analysis. Proceedings of the Society for Information Technology and Teacher Education International Conference, AACE, Chesapeake, 1240-1243.

http://research.educatorscoop.org/SL.IQA.site.final.pdf 
[19] Katz, L. (1972) Developmental Stages of Preschool Teachers. Elementary School Journal, 73, 50-54. http://dx.doi.org/10.1086/460731

[20] Huberman, M., Grounauer, M. and Marti, J. (1993) The Lives of Teachers. New York Teachers College Press, New York, 56-62.

[21] Northcutt, N. and McCoy, D. (2004) Interactive Qualitative Analysis: A Systems Method for Qualitative Research. Sage, Thousand Oaks.

[22] Bann, E.F. (2001) Effects of Media Representations of a Cultural Ideal of Feminine Beauty on Self-Body Image in College-Aged Women: An Interactive Qualitative Analysis. PhD Thesis, The University of Texas at Austin, Austin.

[23] Jonassen, D.H. (1995) Operationalizing Mental Models: Strategies for Assessing Mental Models to Support Meaningful Learning. http://dx.doi.org/10.3115/222020.222166 http://dl.acm.org/citation.cfm?id=222166\&dl=ACM\&coll=DL\&CFID=736878212\&CFTOKEN=22898848

[24] Mancha Jr., G. (2007) Comparing Teacher Perception of English Language Learners at the High School Level: A Case Study of E.P.I.S.D. PhD Thesis, The University of Texas at Austin, Austin.

[25] Hu, H.-J. (2009) An Investigation of User’s Mental Models on Website. Human Centered Design, HCII 2009, LNCS 5619, 722-728. http://dx.doi.org/10.1007/978-3-642-02806-9_84

[26] Bargate, K. (2014) Interactive Qualitative Analysis-A Novel Methodology for Qualitative Research. Mediterranean Journal of Social Science, 5, 11-19. http://dx.doi.org/10.5901/mjss.2014.v5n20p11

[27] Young, M.F. (1998) A Minimax Portfolio Selection Rule with Linear Programming Solution. Management Science, 44, 673-683. http://dx.doi.org/10.1287/mnsc.44.5.673 